\title{
Estudante de Enfermagem em Ensino Clínico: Estudo Qualitativo da Tipologia de Decisão
}

\author{
Fátima Mendes Marques', Maria José Pinheiro¹ e Patricia Vinheira \\ Alves $^{1}$
}

\author{
1 Escola Superior de Enfermagem de Lisboa, Portugal | fmarques@esel.pt; \\ mjpinheiro@esel.pt; palves@esel.pt | https://orcid.org/0000-0001-6581-6711; \\ https://orcid.org/ 0000-0003-1789-5843; https://orcid.org/0000-0003-4705-1721
}

\begin{abstract}
Resumo: A tomada de decisão é uma das competências fundamentais em enfermagem. A aprendizagem da competência de tomada de decisão vem emergindo como área crescente da formação em enfermagem, implicando entre outros aspetos, a reflexão. Os ensinos clínicos são um momento privilegiado de aprendizagem destas competências possibilitando ao estudante um processo de construção do saber profissional, situado em determinado contexto. Com o objetivo de compreender os tipos de tomada de decisão dos estudantes de enfermagem em ensino clínico, optou-se por um estudo de natureza qualitativa, de caracter exploratório e descritivo, com recolha de dados efetuada através de análise documental dos jornais de aprendizagem elaborados pelos estudantes finalistas de enfermagem, em ensino clínico. A análise de conteúdo foi realizada segundo Bardin através do software webQDA®. Participaram no estudo 31 estudantes a frequentarem a Unidade Curricular de Ensino Clínico Cuidar de Clientes com Vulnerabilidade Acrescida e Famílias em Transição que elaboram um total de 31 jornais de aprendizagem. Das 143 unidades de registo identificadas, a Decisão de Intervenção obteve 63 unidades de registo, a Decisão de Compreensão, 41 unidades de registo, a Decisão de Comunicação, 24 unidades de registo, a Decisão de Gestão, 10 unidades de registo, e a Decisão de Temporização e Focalização, 3 e 2 unidades de registo, respetivamente. Conclui-se que os estudantes parecem tomar mais decisões relacionadas com o agir, com a compreensão da situação de cuidados e com a comunicação com o cliente. Com este estudo compreendemos a tipologia da tomada de decisão dos estudantes de enfermagem de forma a potencializar a sua aprendizagem na formação em enfermagem. $\mathrm{O}$ conhecimento que emergiu da interpretação, comparação e síntese dos jornais de aprendizagem, só foi possível pela 'leitura' das narrativas que a metodologia qualitativa viabilizou.
\end{abstract}

Palavras-chave: Estudantes de Enfermagem; Ensino Clínico; Tomada de Decisão; Estudo Qualitativo; Educação em Enfermagem.

The Nursing Student in Clinical Learning: Qualitative Study of the Decision Making Typology

\begin{abstract}
Decision making is a fundamental competency of Nursing. The learning of decision making competence has emerged as an area in development of nursing education, implying, among other aspects, reflection. A privileged moment for learning these competencies, is clinical teaching, enabling the student to construct professional knowledge, situated in context. In order to comprehend the types of decision making of nursing students in clinical education, the method chosen was a qualitative study, of exploratory and descriptive nature, with data collected through documental analysis of the learning journals written in clinical settings, by the finalist nursing students. The content analysis was performed according to Bardin through the software webQDA®. They were 31 students participating in the study, producing 31 learning journals, all were attending the Clinical learning course - Caring for Clients with Increased Vulnerability and Families in Transition. Of the 143 record units identified, the Intervention Decision obtained 63 record units, the Decision of Experimental Understanding 41 record units, the Communication Decision had 24 record units, Service organization, Delivery and Management Decision, 10 record units, and the Timing Decision and Targeting Decision, 3 and 2 record units, respectively. The conclusion was that students seem to make more decisions related to action, understanding of the care situation and communication with the client. With this study we understand the typology of the decision making of the nursing students in order to enhance their learning in nursing education. The knowledge that emerged from the interpretation, comparison and synthesis of the learning journals, was only possible through the 'reading' of the narratives, and the use qualitative methodology.
\end{abstract}

Keywords: Students; Nursing; Clinical Clerkship; Decision Making; Qualitative Study; Education; Nursing. 


\section{Introdução}

A tomada de decisão é uma das competências fundamentais em enfermagem (Baxter \& Boblin, 2008; Marques, 2019). O aumento da acuidade dos clientes, a redução da duração dos internamentos e os constantes avanços da tecnologia são fatores que têm contribuído para a necessidade de os enfermeiros tomarem decisões rapidamente. Os conhecimentos de enfermagem, fundamentais para uma prática baseada em evidência, originam uma adequação das competências de tomada de decisões nos cuidados de saúde. Consequentemente, a formação de enfermagem tem vindo a responsabilizar-se em desenvolver a capacidade dos enfermeiros para tomarem decisões clínicas adequadas (Farčić et al., 2020). A formação inicial em enfermagem visa permitir aos estudantes a aquisição de conhecimentos e competências específicas para que consigam tomar decisões eficazes na prática clínica (Ciftci et al., 2020; Hensel \& Billings, 2020; Tesoro, et al., 2021).

$\mathrm{Na}$ Escola Superior de Enfermagem de Lisboa (ESEL), o Plano de Estudos prevê quatro anos de curso de Licenciatura em Enfermagem: os dois primeiros anos de ensino teórico e teórico-prático e os restantes dois anos de Ensino Clínico (EC). Considerando que o julgamento e a tomada de decisão são mais influenciados pelo background dos enfermeiros do que os dados objetivos sobre a situação de cuidados (Tanner, 2006), a ESEL desenvolveu estratégias de educação para que o estudante adquira essas competências. O Plano de Estudos contempla uma unidade curricular (UC) denominada Processo de Tomada de Decisão em Enfermagem (PTDE) que se centra no desenvolvimento do julgamento clínico e da tomada de decisão, entre outras, e os EC que têm como resultados de aprendizagem a capacitação do estudante para a tomada de decisão em contexto real (Despacho ํㅜ 6687/2017, da Escola Superior de Enfermagem de Lisboa, 2017).

O EC é um momento privilegiado de aprendizagem destas competências (Alarcão \& Rua, 2005; Lam et al., 2020). Os ensinos clínicos ${ }^{1}$ envolvem um processo de construção do saber profissional, situado em determinado contexto, em que o estudante tem como referências o saber teórico em relação com a vivência dos profissionais experientes que os orientam (Alarcão \& Rua, 2005) e a reflexão sobre as suas experiências em contexto da prática. Na procura de maximizar a aprendizagem reflexiva (Bayoumy \& Albeladi, 2020), um dos métodos pedagógicos utilizados é a elaboração do jornal de aprendizagem. Trata-se de um documento escrito no qual o estudante analisa os seus pensamentos, sentimentos, ações/interações num período de tempo, potenciando a sua aprendizagem através da reflexão (González-García et al., 2020)². Face a situações complexas, a reflexão permite ao estudante distinguir os vários fatores que a influenciam e tomar uma decisão ou elaborar um plano de ação (Bulman \& Schutz, 2013).

A tomada de decisão é uma competência que se desenvolve ao longo da formação inicial em enfermagem, sendo uma área relevante na investigação (Bayoumy \& Albeladi, 2020; Farčić et al., 2020; Marino et al., 2020; Phillips et al., 2021; Thompson \& Stapley, 2011). Contudo, com pouca representatividade a nível nacional (Marques, 2019; Marques et al., 2021). Motivo que tem levado a equipa pedagógica da UC PTDE a uma procura de conhecimento sobre os conteúdos lecionados e respetiva aprendizagem pelos estudantes, ao longo da formação inicial em enfermagem.

'Os autores defendem o conceito de 'estágio clínico' como "os momentos de observação e intervenção em contextos de serviços de saúde e afins, com o objectivo de desenvolver capacidades, atitudes e competências" (Alarcão \& Rua, 2005; p. 4). Nesta perspetiva, será utilizado em consonância com o conceito de ensino clínico, ao longo deste trabalho.

2 O jornal de aprendizagem permite a aquisição de competências em enfermagem, a aprendizagem experiencial e sobre si próprio: coping, pensamentos, emoções e sentimentos, além de promover a autoconfiança e a capacidade de empatizar com os clientes (González-García et al., 2020). 
O último ano do curso realizado em contextos de saúde complexos, é representativo de como o estudante lidará com o seu desenvolvimento profissional que começará em breve (González-García et al., 2020; Lam et al., 2021). Neste sentido, foi realizado este estudo com o objetivo de compreender os tipos de tomada de decisão dos estudantes de enfermagem do $4^{\circ}$ ano em ensino clínico de forma a delinear estratégias pedagógicas para facilitar o desenvolvimento de competências. Assim, delineámos como questão de investigação: "Qual a tipologia de decisão que o estudante de enfermagem do $4^{\circ}$ ano do CLE utilizou no ensino clínico?".

\section{Metodologia}

O estudo apresentado é de natureza qualitativa, de caracter exploratório e descritivo, sendo a recolha de dados efetuada através de análise documental dos jornais de aprendizagem elaborados pelos estudantes finalistas de enfermagem, em ensino clínico.

A investigação qualitativa desempenha um papel importante por assegurar uma perspetiva subjetiva das histórias narradas pelos diversos atores sociais (Gonçalves et al., 2021), muito utilizada em profissões de saúde, e especificamente em enfermagem (GonzálezGarcía, et al., 2020; Squires \& Dorsen, 2018). Na procura de compreender a tipologia da tomada de decisão do estudante de enfermagem baseámo-nos num estudo realizado em 2010, com os participantes em contexto clínico semelhante e a mesma referenciação teórica de análise (Marques, 2019) ${ }^{3}$ de modo que as similitudes e as diferenças da experiência pudessem ser identificadas (Squires \& Dorsen, 2018).

A equipa de investigadores foi constituída por 3 professores da equipa docente da UC PTDE da Escola Superior de Enfermagem de Lisboa.

Este estudo teve como participantes os estudantes do $4^{\circ}$ ano do CLE a realizar o ensino clínico Cuidar de Clientes com Vulnerabilidade Acrescida e Famílias em Transição (CCVAFT), do ano letivo 2020-2021, a decorrer entre 4 de janeiro a 23 de abril de 2021. Este ensino clínico contempla três contextos de aprendizagem diferentes: saúde mental e psiquiatria; outras vulnerabilidades; e idoso na comunidade. $O$ jornal de aprendizagem foi um dos documentos a ser elaborado pelos estudantes neste ensino clínico, nos diferentes contextos (ESEL, 2020). Os jornais de aprendizagem foram colhidos entre 18 de janeiro a 13 de março de 2021, através da plataforma de blackboard, validando a autenticidade deste tipo de informação documental (Burgess, 2001).

O critério de seleção dos estudantes foi estarem a frequentar a UC CCVAFT. Como critérios de exclusão: não terem elaborado jornal de aprendizagem no contexto e terem composto jornal de aprendizagem sem referir a tomada de decisão em enfermagem. A amostra corresponde a $13,5 \%$ dos estudantes inscritos no EC CCVAFT (Burgess, 2001). Procurou-se que a amostra obedecesse ao critério de suficiência, pela inclusão e reflexo da variabilidade dos três contextos de ensino clínico e das experiências de aprendizagem, bem como à saturação teórica através da representatividade dos estudantes nos diferentes contextos.

Apesar dos jornais de aprendizagem não terem sido realizados para dar resposta à nossa questão de investigação, o corpus $^{4}$ de analise foi constituído atendendo à representatividade, exaustividade, homogeneidade e relevância dos jornais de aprendizagem em relação ao objeto de estudo. O teor de análise recaiu sobre as perspetivas dos estudantes na tomada de decisão e não sobre os cuidados em si (Bogdan \& Biklen, 2013).

${ }^{3}$ Embora com planos curriculares do CLE diferentes, o EC decorreu no primeiro semestre do $4^{\circ}$ ano do CLE e foi utilizada a tipologia das decisões, segundo Thompson e Staply (2011), em ambos os estudos.

${ }^{4} \mathrm{O}$ corpus "é um conjunto dos documentos tidos em conta para serem submetidos aos procedimentos analíticos" (Bardin, 2016, p. 126). 
A análise de conteúdo foi ancorada em Bardin (2016), tendo-se constituído o corpus de análise, codificado o texto em Unidades de Registo (UR) e agregados os códigos de acordo com a semelhança dos seus sentidos em categorias. Por fim, foram contabilizadas as frequências com foco no tipo de UR e que foi operacionalizada através do software WebQdA® (Costa \& Amado, 2018). Ainda que o programa de análise qualitativa seja uma estratégia facilitadora, este obriga a uma codificação humana (Fornari \& Fonseca, 2020). Razão pela qual, a análise foi conduzida independentemente por dois investigadores e posteriormente procedeu-se a nova triangulação pelo terceiro elemento da equipa de investigação (Denzin \& Lincoln, 2018).

De forma a garantir o rigor do estudo, os procedimentos metodológicos foram permanentemente monitorizados ao longo do mesmo e utilizadas estratégias de autocorreção por parte dos investigadores (Corbin \& Strauss, 2015; Morse et al., 2002).

Este estudo faz parte de uma investigação sobre a aprendizagem da tomada de decisão no processo de cuidados, em contexto clínico, e está aprovada pela Comissão de Ética da ESEL. Os estudantes foram recrutados através de pedido enviado pela plataforma de blackboard, assegurando a confidencialidade e garantia de ausência de coerção e consequências prejudiciais, tendo o consentimento sido enviado por e-mail. Ao longo de todo o processo de investigação foram eliminados os nomes dos estudantes dos jornais de aprendizagem e foram identificados com $E$ (estudante) e adicionado um número pela ordem como foram recebidos os consentimentos informados. Complementou-se com a caracterização do género através das letras $F$ (Feminino) e M (Masculino).

\section{Resultados}

Participaram no estudo 31 estudantes, com um total de 31 jornais de aprendizagem. 0 género predominante era o feminino com 90,3\%, e a idade variou entre 21 e 33 anos, sendo a média de 22,45 anos.

A análise da tipologia da tomada de decisão que os estudantes narraram nos seus registos foi suportada pela categorização desenvolvida por Thompson e Stapley (2011) (Tabela 1). Os autores descrevem seis tipos de tomada de decisão (intervenção, focalização, temporização, comunicação, gestão e compreensão) que foram descritos nos jornais de aprendizagem.

Tabela 1. Tipos de decisões, segundo Thompson e Stapley.

\begin{tabular}{|c|c|c|}
\hline Tipo de decisão & Definição & Exemplo \\
\hline $\begin{array}{l}\text { Decisão } \\
\text { (intervenção) }\end{array}$ & $\begin{array}{l}\text { Selecionar entre } \\
\text { intervenções. }\end{array}$ & $\begin{array}{l}\text { A escolha de um colchão para um idoso } \\
\text { que tenha sido admitido com uma } \\
\text { obstrução intestinal aguda. }\end{array}$ \\
\hline $\begin{array}{l}\text { Decisão } \\
\text { (focalização) }\end{array}$ & $\begin{array}{l}\text { Selecionar qual o cliente que } \\
\text { beneficiará mais com a } \\
\text { intervenção de enfermagem. }\end{array}$ & $\begin{array}{l}\text { Decidir quais os clientes que deveriam ter } \\
\text { meias anti-embólicas. }\end{array}$ \\
\hline $\begin{array}{l}\text { Decisão } \\
\text { (temporização) }\end{array}$ & $\begin{array}{l}\text { Selecionar a melhor altura } \\
\text { para intervir. }\end{array}$ & $\begin{array}{l}\text { Decidir a altura para começar a educação } \\
\text { para a saúde sobre asma a clientes com } \\
\text { asma recém-diagnosticados. }\end{array}$ \\
\hline $\begin{array}{l}\text { Decisão } \\
\text { (comunicação) }\end{array}$ & $\begin{array}{l}\text { Selecionar qual a informação } \\
\text { a recolher e a transmitir aos } \\
\text { clientes, familiares e equipa } \\
\text { de enfermagem. }\end{array}$ & $\begin{array}{l}\text { Decidir como abordar a reabilitação } \\
\text { cardíaca depois de um enfarte agudo do } \\
\text { miocárdio num cliente idoso que vive } \\
\text { sozinho, apesar de ter familiares que } \\
\text { vivam próximo }\end{array}$ \\
\hline Decisão (gestão) & $\begin{array}{l}\text { Definir ou processar a } \\
\text { transferência de cuidados. }\end{array}$ & $\begin{array}{l}\text { Decidir como organizar a transferência, } \\
\text { para que a comunicação seja mais eficaz. }\end{array}$ \\
\hline $\begin{array}{l}\text { Decisão } \\
\text { (compreensão) }\end{array}$ & $\begin{array}{l}\text { Interpretar sinais no processo } \\
\text { de cuidados. }\end{array}$ & $\begin{array}{l}\text { Decidir como tranquilizar um cliente que } \\
\text { esteja preocupado com uma possível } \\
\text { paragem cardíaca depois de testemunhar } \\
\text { uma paragem noutro cliente. }\end{array}$ \\
\hline
\end{tabular}

Fonte: Traduzido e adaptado de Thompson e Stapley (2011). 
Da análise efetuada, entre as 143 unidades de registo identificadas, a Decisão de Intervenção foi a que obteve maior frequência, com 63 unidades de registo, seguida da Decisão de Compreensão, com 41 unidades de registo, da Decisão de Comunicação, com 24 unidades de registo, da Decisão de Gestão, com 10 unidades de registo, e por fim da Decisão de Temporização e a de Focalização, com 3 e 2 unidades de registo, respetivamente (Tabela 2).

Tabela 2. Tipos de tomada de decisão dos estudantes, segundo Thompson e Stapley.

\begin{tabular}{lcc}
\hline \multicolumn{1}{c}{ Tipo de decisão } & $\begin{array}{c}\text { Frequência } \\
\text { por categoria }\end{array}$ & Total de unidades de registo \\
\hline Decisão (intervenção) & 63 & \\
Decisão (focalização) & 2 & $\mathbf{1 4 3}$ \\
Decisão (temporização) & 3 & \\
Decisão (comunicação) & 24 & \\
Decisão (gestão) & 10 & \\
Decisão (compreensão) & 41 & \\
\hline
\end{tabular}

Na categoria Decisão de Intervenção os estudantes parecem descrever como decidem intervir pela identificação do risco para eles e para o cliente:

Levei a cliente na cadeira de rodas para o pé do leito, onde era mais seguro e onde ela se poderia segurar caso alguma coisa acontecesse (E21-M).

Sobre a categoria Decisão de Compreensão, as narrativas dos estudantes sugerem uma interpretação do processo de cuidados que os leva a decidir:

Dado o crescente estado de ansiedade e inquietação do utente perante esta situação, eu e a minha enfermeira respeitámos, naquele momento, a sua decisão (E11-F).

Relativamente à categoria Decisão de Comunicação, na seleção da informação a transmitir aos clientes e família, os estudantes parecem facilitar a cooperação e a interação entre eles:

Adotei ainda a técnica da reformulação, de forma a esclarecer e facilitar a compreensão, bem como a técnica da clarificação, tentando clarificar o que foi dito anteriormente (E24-F).

Na categoria Decisão de Gestão, o processar da transferência de cuidados sugere estar presente entre estudantes de enfermagem, como forma de assegurar a sua continuidade:

Pedi a uma estudante de enfermagem que também se encontrava lá, para que ficasse de vigilância na M.R., de forma discreta (E4-F).

Os resultados da categoria Decisão de Temporização, sugerem uma decisão baseada na seleção da melhor ocasião para intervir:

O senhor não era o único pela qual estava responsável, pelo que tive de gerir o tempo para prestar os melhores cuidados dentro das minhas capacidades a todos os clientes (E5-F).

A Decisão de Focalização parece implicar uma intervenção de enfermagem centrada na pessoa alvo dos cuidados:

Procurei ser imparcial e realizar as minhas tarefas de forma completa, indo de encontro às necessidades dos meus outros clientes sempre mantendo a segurança e qualidade dos mesmos (E6-F). 


\section{Discussão}

Os estudantes de enfermagem devem aprender a tomar decisões de forma independente e autónoma (Tesoro et al., 2021), processo em que o ensino clínico se assume como um momento privilegiado de aprendizagem (Ciftci et al., 2020). No EC CCVAFT, os estudantes de enfermagem parecem tomar mais decisões relacionadas com o agir, com a compreensão da situação de cuidados e com a comunicação com o cliente.

Nas narrativas dos estudantes a Decisão de Intervenção emergiu com maior destaque em que o estudante concebe hipóteses face à resolução do problema e seleciona as apropriadas à situação e ao contexto (Marques et al., 2021). A tomada de decisão parece depender da situação com que se confrontam, na procura de corresponder às necessidades individuais dos clientes (Phillips et al., 2021; Thompson \& Stapley, 2011). Comparativamente ao estudo de Marques (2019), a decisão de intervenção surgiu em terceiro lugar na referenciação dos estudantes. Equacionamos se esta diferença se deve à redução de uma semana no $E C$ e às interrupções para isolamentos profiláticos e infeções por COVID-19 condicionando a tomada de decisão do estudante de acordo de contaminação (Baxter \& Boblin, 2008; Soccol et al., 2020), aspeto que carece de investigação.

A Decisão de Compreensão surge igualmente com notabilidade no modo como os estudantes tomavam decisões. O processo de reflexão que subsidia 0 ensino de enfermagem, quer na componente teórica, quer na prática, parece ter permitido aos estudantes analisar o seu processo de raciocínio durante os cuidados ao cliente e, simultaneamente, torná-los conscientes desse processo reflexivo (Ciftci et al., 2020). Em analogia com o estudo que nos serviu de suporte (Marques, 2019), manteve-se a metacognição como elemento crucial na tomada de decisão, contribuindo para a compreensão do processo de cuidados (Bektas et al., 2020). Os métodos de raciocínio envolvidos na análise crítica e interpretação dos dados, parecem ter contribuído para a 'leitura' do cliente, dos acontecimentos e das relações estabelecidas (Tanner, 2006).

Distintivamente, também a Decisão de Comunicação emergiu face às restantes tipologias de tomada de decisão dos estudantes. O conhecimento subjacente na orientação da comunicação (Tesoro et al., 2021), bem como o desenvolvimento pessoal e profissional adequado parece levar o estudante a comunicar com confiança (González-García et al., 2020) com o cliente numa tentativa de o envolver no processo de decisão. Relativamente aos resultados apresentados por Marques (2019), a seleção do que comunicar ao cliente e família e à equipa de enfermagem foi a decisão com maior referenciação. Atualmente, 0 uso de equipamentos de proteção individual e a manutenção do distanciamento social, que implicações terá na forma como estudante interagiu com o cliente e família? (Soccol et al., 2020).

A Decisão de Gestão, com menor representatividade que as anteriores, surge associada ao processo de organização e gestão de serviços pelos estudantes de enfermagem. 0 seu processo de decisão sugere o uso de recursos como os seus pares, e outros profissionais de saúde, para a continuidade dos cuidados (Baxter \& Boblin, 2008; Thompson \& Stapley, 2011). Um dos aspetos a destacar foi a inexistência de referenciações nesta categoria no estudo anterior (Marques, 2019). Será que a UC PTDE permitiu aos estudantes sentirem-se mais confiantes na forma como tomavam decisões (Phillips et al., 2021) sobre a organização e articulação dos cuidados?

Escassamente, os estudantes atribuíram a Decisão de Temporização, ou seja, a seleção da melhor altura para intervir (Thompson \& Staply, 2011). Na adequação e priorização dos cuidados, os estudantes sugerem demonstrar um raciocínio clínico que permite uma tomada de decisão eficaz de acordo com a compreensão da situação clínica (Bektas et al., 2020). Esta referenciação teve um resultado semelhante no estudo anterior (Marques, 2019), ficando em penúltimo na análise desenvolvida. Será que os estudantes são preparados adequadamente ao longo da sua formação para este tipo de decisão? (KlenkeBorgmann et al, 2021; Phillips et al., 2021). 
A menor relevância do tipo de decisões que os estudantes tomavam recaiu na Decisão de Focalização. O estudante parece decidir sobre a intervenção a realizar na procura do maior benefício para o cliente (Thompson \& Staply, 2011), implicando um conhecimento deste, quer pela forma como frequentemente reage às situações de cuidado, quer enquanto pessoa (Tanner, 2006). Também no estudo de Marques (2019), decidir qual o cliente que beneficiava mais com a intervenção de enfermagem, surgiu por último. Perante as situações adversas que a pandemia trouxe para as instituições de saúde, de que modo os estudantes foram capazes de tomar decisões com base nas necessidades individuais dos clientes? (Phillips et al., 2021).

Relativamente às limitações do estudo, e tendo em conta a metodologia utilizada, estes resultados não se podem extrapolar. Este estudo podia ser subsidiado com triangulação de técnicas de colheita de dados como entrevistas ou focus grupo. Outra limitação incide na elaboração dos jornais de aprendizagem não ter o intuito de objetivar esta investigação, no entanto, a riqueza dos documentos permitiu-nos constituir um corpus cuja análise dá resposta à questão de investigação e ao objetivo do estudo.

\section{Conclusões}

A tomada de decisão em ensino clínico que emerge dos relatos dos estudantes sugere uma valorização da decisão de agir, seguida da de compreensão da situação de cuidados e da comunicação com o cliente. Com menor impacto surgem a tomada de decisão de gestão para assegurar a continuidade de cuidados, de temporização na escolha do melhor momento para atuar e de focalização, ou seja, a seleção da intervenção com maior benefício para o cliente. A metodologia qualitativa pela interpretação, comparação e síntese da 'leitura' das narrativas que os jornais de aprendizagem representaram, permitiu uma compreensão da tomada de decisão do estudante de enfermagem.

Como implicação ao nível da formação, a compreensão dos vários tipos de tomada de decisão do estudante de enfermagem permite à equipa docente da UC PTDE refletir e equacionar os conteúdos e as metodologias pedagógicas de modo a potenciar o desenvolvimento de todos os tipos de tomada de decisão dos estudantes no seu percurso académico. É nossa intenção divulgar junto da comunidade académica os resultados obtidos nesta investigação no sentido de potenciar outras estratégias pedagógicas que promovam o desenvolvimento da tomada de decisão dos estudantes.

Ao nível de investigação, com este estudo compreendemos o tipo tomada de decisão dos estudantes de enfermagem participantes em EC. Concomitantemente, os resultados e limitações desta investigação deixam em aberto outras áreas de estudo sobre a tomada de decisão dos estudantes de enfermagem.

\section{Referências}

Alarcão, I., \& Rua, M. (2005). Interdisciplinaridade, estágios clínicos e desenvolvimento de competências. Texto \& Contexto - Enfermagem, 14(3), 37382. https://doi.org/10.1590/S0104-07072005000300008

Bardin, L. (2016). Análise de conteúdo. Edições 70.

Baxter, P. E., \& Boblin, S. (2008). Decision making by baccalaureate nursing students in the clinical setting. Journal of Nursing Education, 47(8), 345-50. https://doi.org/10.3928/0148483420080801-02

Bayoumy, H. M. M., \& Albeladi, G. A. (2020). Clinical judgment skills among junior-level nursing students enrolled in adult health nursing courses: Errors and risk level classification. Nurse Education in Practice, 48, 102888. https://doi.org/10.1016/j.nepr.2020.102888

Bektas, I., Bektas, M., Ayar, D., Akdeniz Kudubes, A., Sal, S., Selekoglu OK, Y., \& Celik, I. (2020). The predict of metacognitive awareness of nursing students on self-confidence and anxiety in clinical decision-making. Perspectives in Psychiatric Care, 1-6. https://doi.org/10.1111/ppc.12609 
Bogdan, R., \& Biklen, S. (2013). Investigação qualitativa em educação.(2 ${ }^{\mathrm{a}}$ ed.). Porto Editora.

Bulman, C., \& Schutz, S. (2013). Reflective practice in nursing. (5 $5^{\text {th }}$ ed.). John Wiley \& Sons.

Burgess, R. C. (2001). A pesquisa de terreno: uma introdução. Celta Editora.

Ciftci, B., Gok, S., Aksoy, M., \& Avsar, G. (2020). The effect of internships on clinical decision making and professional values of nursing students. International Journal of Caring Sciences, 13(2), 1230-39. http://www.internationaljournalofcaringsciences.org/docs/47_1_ciftci_original_13_2.pdf

Corbin, J. \& Strauss, A. (2015). Basics of qualitative research. ( $4^{\text {th }}$ ed.). SAGE Publications, Inc.

Costa, A. P., \& Amado, J. (2018). Análise de conteúdo suportada por software. Ludomedia.

Denzin, N., \& Lincoln, Y. S. (2018). The Sage handbook of qualitative research. $\left(5^{\text {th }}\right.$ ed.). SAGE Publications, Inc.

Despacho n. ${ }^{\circ} 6687 / 2017$ da Escola Superior de Enfermagem de Lisboa. (2017). Diário da

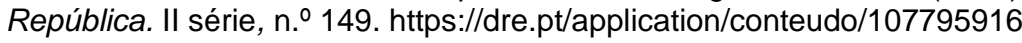

Escola Superior de Enfermagem de Lisboa (ESEL). (2020). Guia orientador da unidade curricular ensino clínico: Cuidar de Clientes com Vulnerabilidade Acrescida e Famílias em Transição. ESEL

Farčić, N., Barać, I., Lovrić, R., Pačarić, S., Gvozdanović, Z., \& llakovac, V. (2020). The influence of self-concept on clinical decision-making in nurses and nursing students: A cross-sectional study. International journal of environmental research and public health, 17(9), 3059. https://doi.org/10.3390/ijerph17093059

Fornari, L. F., \& Fonseca, R. (2020). Critical-emancipatory workshop analysis through qualitative analysis software. The Qualitative Report, 25(13), 90-103. https://nsuworks.nova.edu/tqr/vol25/iss13/7

Gonçalves, J. P., Marques, C. G., \& Gonçalves, S. P. (2021). Manual de investigação qualitativa conceção, análise e aplicações. Pactor

González-García, M., Lana, A., Zurrón-Madera, P., Valcárcel-Álvarez, Y., \& Fernández-Feito, A. (2020). Nursing students' experiences of clinical practices in emergency and intensive care units. International Journal of Environmental Research and Public Health, 17(16), 5686. https://doi.org/10.3390/ijerph17165686

Hensel, D., \& Billings, D. M. (2020). Strategies to teach the National Council of state boards of nursing clinical judgment model. Nurse Educator, 45(3), 128-32. https://doi.org/10.1097/NNE.0000000000000773

Klenke-Borgmann, L., Cantrell, M. A., \& Mariani, B. (2021). Clinical judgment in nursing students after observation of in-class simulations. Clinical Simulation in Nursing, 51, 19-27. https://doi.org/10.1016/j.ecns.2020.11.006

Lam, C. K., Schubert, C. F., \& Herron, E. K. (2020). Evidence-based practice competence in nursing students preparing to transition to practice. Worldviews on Evidence-Based Nursing, 17(6), 418-26. https://doi.org/10.1111/wvn.12479

Marino, M. A., Andrews, K., \& Ward, J. (2020). Clinical decision making at the bedside. Nursing Clinics, 55(1), 29-37. https://doi.org/10.1016/j.cnur.2019.10.003

Marques, F. M., David, C., dos Santos, A. F., Neves, S., Pinheiro, M. J., \& Leal, M. T. (2021). Perceptions of senior nursing students regarding clinical decision-making. Rev Bras Enferm,74(1), e20200921. http://dx.doi.org/10.1590/0034-7167-2020-0921

Marques, M. F. M. (2019). Decision making from the perspective of nursing students. Rev Bras Enferm, 72(4), 1102-8. http://dx.doi.org/10.1590/0034-7167-2018-0311

Morse, J., Barrett, M., Mayan, M., Olson, K. \& Spiers, J. (2002). Verification strategies for establishing reliability and validity in qualitative research. International Journal of Qualitative Methods, 1 (2), 13-22. https://doi.org/10.1177/160940690200100202

Phillips, B. C., Morin, K., \& Valiga, T. M. (2021). Clinical decision making in undergraduate nursing students: A mixed methods multisite study. Nurse Education Today, 97, 104676. https://doi.org/10.1016/j.nedt.2020.104676 
Rodríguez-García, M. C., Gutiérrez-Puertas, L., Granados-Gámez, G., Aguilera-Manrique, G., \& Márquez-Hernández, V. V. (2021). The connection of the clinical learning environment and supervision of nursing students with student satisfaction and future intention to work in clinica placement hospitals. Journal of Clinical Nursing, 1-9. https://doi.org/10.1111/jocn.15642

Soccol, K., dos Santos, N., \& Marchiori, M. (2020). Estágio curricular supervisionado no contexto da COVID-19 e o desenvolvimento profissional de estudantes de Enfermagem. Enfermagem em Foco, 11(2.ESP). https://doi.org/10.21675/2357-707X.2020.v11.n2.ESP.4173

Squires, A., \& Dorsen, C. (2018). Qualitative research in nursing and health professions regulation. Journal of Nursing Regulation, 9(3), 15-26. https://doi.org/10.1016/S21558256(18)30150-9

Tanner, C. A. (2006). Thinking like a nurse: a research-based model of clinical judgment in nursing. The Journal of Nursing Education, 45(6), 204-11. https://doi.org/10.3928/0148483420060601-04

Tesoro, M. G., Simmons, A. M., Barros, A. L. B. L., Lopes, C. T., Guandalini, L. S., Cruz, E. D. D. A., \& Maurício, A. B. (2021). Effects of clinical reasoning prompts on nursing students' clinical judgment for a patient experiencing respiratory distress. International Journal of Nursing Knowledge, 32(1), 37-43. https://doi.org/10.1111/2047-3095.12286

Thompson, C., \& Stapley, S. (2011). Do educational interventions improve nurses' clinical decision making and judgement? A systematic review. International Journal of Nursing Studies, 48(7), 881-93. https://doi.org/10.1016/j.ijnurstu.2010.12.005 